\title{
The (Non)Automaticity of Amygdala Responses to Threat: On the Issue of Fast Signals and Slow Measures
}

\author{
Tobias Brosch ${ }^{1}$ and Matthias J. Wieser ${ }^{1,2}$ \\ ${ }^{1}$ Department of Psychology, New York University, New York, New York 10003, and ²University of Würzburg, 97070 Würzburg, Germany \\ Review of Mothes-Lasch et al.
}

A huge amount of sensory information continually enters the brain via multiple channels. Due to capacity limits, the brain must select a subset of information for more thorough processing. Voluntary topdown attention mechanisms, mediated by a frontoparietal attention network, boost the processing of stimuli in line with the current expectations and behavioral goals of the organism. Complementing this controlled mechanism, emotional-especially threatening — stimuli are prioritized automatically, as demonstrated across many experimental paradigms such as visual search, interference, and spatial cueing tasks. At the neural level, these effects have been linked to the amygdala, which is thought to detect threatening stimuli and boost their processing via feedback projections to sensory cortex and frontoparietal attention regions (LeDoux, 1996).

It has been suggested that threat detection by the amygdala occurs automatically, in the sense that it occurs independent of attentional resources. However, this claim has been debated widely in the literature and has spurred several fMRI studies investigating whether increased amygdala activation to threat may indeed occur independently

Received Aug. 9, 2011; revised Aug. 24, 2011; accepted Aug. 26, 2011.

This work was supported by Swiss National Science Foundation Grant PA00P1_131435 to T.B. We thank Andreas Keil and Erno Hermans for helpful comments.

Correspondence should be addressed to Tobias Brosch, Department of Psychology, New York University, 6 Washington Place, New York, New York 10003. E-mail: tobias.brosch@nyu.edu.

DOI:10.1523/JNEUROSCI.4089-11.2011

Copyright $\odot 2011$ the authors $\quad 0270-6474 / 11 / 3114451-02 \$ 15.00 / 0$ of processing resources and task demands. Such studies typically manipulate voluntary attention and measure the effect of this manipulation on the amygdala response to threat. Conflicting results have been reported. In some studies, enhanced amygdala responses to emotional relative to neutral stimuli have been found to arise even when attention is guided away from the stimuli (Vuilleumier et al., 2001). In contrast, other studies have reported reduced or abolished amygdala responses to threat stimuli presented under high attentional load (Pessoa et al., 2002).

Up until now, research on emotionattention interactions has manipulated attention mainly within a sensory modality, e.g., by directing visuospatial attention toward or away from visual information such as angry faces, or directing auditory attention toward or away from angry prosody. However, in real-life situations, humans typically encounter and simultaneously attend to input through multiple senses (such as vision, audition, olfaction, and touch), and any of these modalities may convey information about threatening events. If the automatic prioritization of emotional information is a general principle of functional brain organization, emotional information should also increase amygdala activation when attention is voluntarily focused on a different sensory modality.

A recent article by Mothes-Lasch and colleagues (2011) addressed this issue. The authors presented either angry or neutral prosody to their participants, while also presenting visual information (either a white cross or a circle). Participants had to attend either to the auditory information (making gender judgments) or to the visual information (indicating which stimulus they were seeing). Previous within-modality studies had observed increased BOLD signal toward angry prosody in amygdala and superior temporal cortex independent of the focus of voluntary attention. In contrast, the between-modality attention manipulation used by Mothes-Lasch and colleagues (2011) had a striking effect on the BOLD signal: significantly higher BOLD signal in bilateral superior temporal cortex, left amygdala, and left insula was observed for angry compared with neutral prosody only when participants attended to the speech while making gender judgments. In contrast, no increased BOLD signal to angry prosody was observed when participants were attending to the visual modality. The authors interpret their data as indicating that enhanced activation of amygdala and auditory cortex to threat-related voices depends on modality-specific attention.

With their study, Mothes-Lasch and colleagues (2011) extend the automaticity debate to the cross-modal level. This is the necessary next step for the field, and their findings may have important implications for our understanding of the emotional brain. If the brain automatically prioritizes emotional information, the mechanism responsible should operate supramodally, because threatening information may be conveyed in any modality. The demonstration that voluntary attention needs to be 
allocated toward the modality in which emotional information is conveyed in order for that information to activate the amygdala makes a very strong case against suggestions of automatic threat processing.

In contrast to the results of MothesLasch et al. (2011), however, other recent studies have found cross-modal effects of emotional stimuli, demonstrating that emotional auditory information can facilitate identification of visual targets (Zeelenberg and Bocanegra, 2010) and boost early brain responses toward visual targets (Brosch et al., 2009) when participants' attention was directed toward the visual modality. In addition, visual emotional information has been shown to reduce resource allocation to an auditory startle probe, as indicated by a decreased amplitude of the attention-sensitive P3 component of the electroencephalogram (Keil et al., 2007). Thus, studies using behavioral and electrophysiological measures have provided at least indirect evidence for the prioritization of emotional information that is presented outside the attended modality.

We would like to suggest a potential alternative explanation for the absence of emotional effects in the BOLD signal in the visual attention condition in MothesLasch et al. (2011). Data obtained with fMRI measurements have a relatively limited temporal resolution due to the sluggishness of the BOLD response, and events occurring during a temporal window of $\sim 2 \mathrm{~s}$ are usually averaged together. But two recent studies using methods with temporal resolution in the millisecond range (intracranial EEG and MEG) have revealed dissociations between a very rapid amygdala response to threatening faces that was independent of voluntary attention and a later response that interacted with attention (Luo et al., 2010; Pourtois et al., 2010). Pourtois and colleagues (2010) measured intracranial local field potentials (which correlate with BOLD responses, but have higher temporal resolution) in a patient with depth electrodes implanted in the lateral amygdala, while attention was directed either toward or away from threatening or neutral faces. A rapid (140-290 ms) differential response to the emotionality of the faces was detected in the lateral amygdala, independent of whether attention was directed toward or away from the faces. A later (starting at $700 \mathrm{~ms}$ ) response to emotional stimuli interacted with attention, in that activation differences between threatening and neutral faces were only detected when the faces were in the focus of voluntary attention. Similarly, Luo and colleagues (2010) used MEG to measure gamma band activity within the amygdala during the processing of threatening versus neutral faces both under high and low attentional task load. They observed a very rapid increase in gamma power after the onset of threatening faces (30-60 ms) independent of task load. Later (at 280$340 \mathrm{~ms}$ ), they observed an interaction of emotion with their attention manipulation, in that significantly greater gamma band activity in response to threatening expressions occurred within the amygdala under low-load conditions, whereas no such difference was observed under high-load conditions.

These two studies provide evidence for an early amygdala response to threatening faces that is automatic in the sense that it arises regardless of task load or attentional focus. In contrast, at a slightly later time, the amygdala response depended on attention, such that higher activation to threatening faces was abolished by high task load or a manipulation of spatial attention away from the face. Importantly, both the early automatic and the later nonautomatic amygdala response lie within the time window of one volume acquisition in fMRI studies. The early response might thus be blurred and not detected by the slow BOLD signal, which integrates over both responses. In this context, it is possible that different experimental designs or different recording parameters may differentially bias the sensitivity of fMRI BOLD measures toward the early or late phases of amygdala activation. For example, many of the fMRI studies reporting automaticity of amygdala activa- tion used event-related designs (Vuilleumier et al., 2001), whereas many of the studies reporting a modulation by attention (Pessoa et al., 2002; Mothes-Lasch et al., 2011) used block designs.

To sum up, Mothes-Lasch and colleagues (2011) provided important evidence for a role of cross-modal attention in modulating the amygdala response to emotional prosody. Due to temporal limitations of the standard fMRI signal, however, it remains an interesting challenge for future research to disentangle the roles of very rapid, potentially automatic amygdala responses and later stages of amygdala activity, by using more time-sensitive methods like EEG, MEG, or very rapid fMRI sequences that have a higher probability of picking up rapid amygdala responses.

\section{References}

Brosch T, Grandjean D, Sander D, Scherer KR (2009) Cross-modal emotional attention: emotional voices modulate early stages of visual processing. J Cogn Neurosci 21:1670-1679.

Keil A, Bradley MM, Junghöfer M, Russmann T, Lowenthal W, Lang PJ (2007) Cross-modal attention capture by affective stimuli: evidence from event-related potentials. Cogn Affect Behav Neurosci 7:18-24.

LeDoux JE (1996) The emotional brain. New York: Simon and Shuster.

Luo Q, Holroyd T, Majestic C, Cheng X, Schechter J, Blair RJ (2010) Emotional automaticity is a matter of timing. J Neurosci 30:5825-5829.

Mothes-Lasch M, Mentzel HJ, Miltner WH, Straube T (2011) Visual attention modulates brain activation to angry voices. J Neurosci 31:9594-9598.

Pessoa L, McKenna M, Gutierrez E, Ungerleider LG (2002) Neural processing of emotional faces requires attention. Proc Natl Acad Sci U S A 99:11458-11463.

Pourtois G, Spinelli L, Seeck M, Vuilleumier P (2010) Temporal precedence of emotion over attention modulations in the lateral amygdala: intracranial ERP evidence from a patient with temporal lobe epilepsy. Cogn Affect Behav Neurosci 10:83-93.

Vuilleumier P, Armony JL, Driver J, Dolan RJ (2001) Effects of attention and emotion on face processing in the human brain: an eventrelated fMRI study. Neuron 30:829-841.

Zeelenberg R, Bocanegra BR (2010) Auditory emotional cues enhance visual perception. Cognition 115:202-206. 